Pacific Journal of Mathematics

A FUNCTION-THEORETIC APPROACH TO THE STUDY O 


\title{
A FUNCTION-THEORETIC APPROACH TO THE STUDY OF NONLINEAR RECURRING SEQUENCES
}

\author{
J. N. Franklin and S. W. Golomb
}

For every real $r \geqq 0$, there is a sequence $\left\{b_{n}^{(r)}\right\}$ defined by

$$
b_{0}^{(r)}=1, \quad b_{n+1}^{(r)}=\prod_{i=1}^{n} b_{i}^{(r)}+r \text { for } n \geqq 0 .
$$

These sequences were considered previously, in [1], for integer values of $r$, and it was shown that there is a constant $\theta=\theta(r)$ such that

$$
b_{n+1}^{(r)} \sim \theta^{2^{n}}, \quad n \rightarrow \infty
$$

for each $r=1,2,3, \cdots$. It was observed that

$$
b_{n+1}^{(2)}=2^{2^{n}}+1, \quad n \geqq 0,
$$

whereby $\theta(2)=2$, and the problem was proposed "to determine the algebraic or transcendental character of the real numbers $\theta(r)$ for $r=1,3,4,5,6, \cdots$."

In this paper, we observe explicitly (in $\S I I)$ that

$$
b_{n}^{(4)}=\tau^{2^{n}}+\tau^{-2^{n}}+2, \quad n \geqq 1,
$$

where $\tau=(\sqrt{5}+1) / 2=1.618 \cdots$ is the "Golden Mean", and thus $\theta(4)=\tau^{2}=(\sqrt{5}+3) / 2=2.618 \cdots$.

Moreover, we extend the result (2) by showing, for every real $r>0$, there is a real constant $\theta=\theta(r)>1$ such that

$$
b_{n+1}^{(r)}=\theta^{2^{n}}+\frac{r}{2}+\frac{1}{8} r(r-2) \theta^{-2^{n}}+O\left(\theta^{-2^{n+2}}\right), \quad n \rightarrow \infty .
$$

Thus for $r \neq 2$ the sequence $\left\{\beta_{n}^{(r)}\right\}=\left\{b_{n}^{(r)}-(r / 2)\right\}$ differs from the sequence $\left\{\theta^{2^{n-1}}(r)\right\}$ by an amount which approaches 0 exponentially as $n \rightarrow \infty$. The case $r=4$, described in (4), is illustrative of this behavior, while the case $r=2$, described in (3), is exceptional in that the error term is identically 0 .

For $r=0, b_{n}^{(0)}=\beta_{n}^{(0)}=1$ for all $n \geqq 0$, so that $\theta(0)=1$; and $\theta(r)$ is a continuous, monotone increasing function of $r \geqq 0$.

The basic tool used in treating the general case is a new theorem in function theory ( $\S$ III), which is ideally suited to the study of sequences 
generated by nonlinear (and especially polynomial) recursions. The function-theoretic approach also reveals $(\S \mathrm{V})$ an unexpected connection between the class of nonlinear recursions considered here, and the enumeration problem for rooted trees.

II. The case $r=4$. We observe that $\tau^{2}=(3+\sqrt{5}) / 2$ and $\tau^{-2}=(3-\sqrt{5}) / 2$. Thus $\tau^{2^{1}}+\tau^{-2^{1}}=3=b_{1}^{(4)}-2=\beta_{1}^{(4)}$. Setting $r=4$ in (1), we see

$$
b_{n+1}^{(4)}=b_{n}^{(4)} \cdot \prod_{i=1}^{n-1} b_{i}^{(4)}+4=b_{n}^{(4)}\left(b_{n}^{(4)}-4\right)+4=\left(b_{n}^{(4)}-2\right)^{2} .
$$

The general identity

$$
b_{n}^{(4)}=\tau^{2^{n}}+\tau^{-2^{n}}+2, \quad n \geqq 1
$$

is established inductively by

$$
b_{n+1}^{(4)}=\left(b_{n}^{(4)}-2\right)^{2}=\left(\tau^{2^{n}}+\tau^{-2^{n}}\right)^{2}=\tau^{2^{n+1}}+\tau^{-2^{n+1}}+2,
$$

since we have already verified (6) for the case $n=1$.

III. A function-theoretic approach. The behavior of $b_{n}^{(r)}$ as $n \rightarrow \infty$ will be elucidated by means of the following.

THEOREM. For $|z| \geqq R>0$ let $p(z)$ be analytic, and let $|p(z)| \geqq$ $A|z|$, where $A>1$. Then there is a unique positive integer, $m$, a unique complex constant, $c \neq 0$, and a unique function, $f(z)$, analytic and nonzero for $|z|>R$, such that $f(z) \cong z$ as $z \rightarrow \infty$, and such that

$$
f(p(z)) \equiv c\{f(z)\}^{m} .
$$

Proof. Since $p(z) \rightarrow \infty$ as $z \rightarrow \infty$, this function has a pole at infinity. If the pole is of order $M$ :

$$
p(z) \cong C z^{M} \quad \text { as } \quad z \rightarrow \infty
$$

and if a functional equation of the form (8) holds, then since $f(z)$ is required to be $\cong z$ at $\infty$, we conclude that $c=C$ and $m=M$.

Let the unknown function $f(z)$ be represented by $f(z)=z \exp \varphi(z)$ where $\varphi(z) \rightarrow 0$ as $z \rightarrow \infty$. The functional equation (8) now takes the form

$$
p(z) \exp \varphi(p(z))=c z^{m} \exp [m \varphi(z)]
$$

which is true if 


$$
\varphi(z)=q(z)+\frac{1}{m} \varphi(p(z))
$$

where $m q(z)$ is the logarithm of $p(z) /\left(c z^{m}\right)$ which tends to zero as $z \rightarrow \infty$.

A solution of the functional equation (9) can be found by the method of iteration. Let $\varphi_{0}(z)=q(z)$, and define $\varphi_{1}(z), \varphi_{2}(z), \cdots$ by

$$
\varphi_{n+1}(z)=q(z)+\frac{1}{m} \varphi_{n}(p(z)) \quad(n=0,1, \cdots) .
$$

Since $|p(z)| \geqq A|z|>|z|$, all the functions $\varphi_{n}(z)$ are analytic for $|z| \geqq R$. Since $q(z)$ is regular and equal to zero at $z=\infty$, there is a constant $Q$ such that

$$
\left|\varphi_{0}(z)\right|=|q(z)| \leqq Q /|z| \quad(|z| \geqq R) .
$$

We can now prove by induction that, for $n=1,2, \cdots$,

$$
\left|\varphi_{n}(z)-\varphi_{n-1}(z)\right| \leqq Q /\left(A^{n}|z|\right) .
$$

For $n=1$, we deduce from (10) and (11)

$$
\left|\varphi_{1}(z)-\varphi_{0}(z)\right|=\frac{1}{m}\left|\varphi_{0}(p(z))\right| \leqq \frac{1}{m} Q /|p(z)| \leqq Q /(A|z|) .
$$

For $n>1$, we have

$$
\varphi_{n+1}(z)-\varphi_{n}(z)=\frac{1}{m}\left\{\varphi_{n}(p(z))-\varphi_{n-1}(p(z))\right\}
$$

Assuming (12), we find

$$
\left|\varphi_{n+1}(z)-\varphi_{n}(z)\right| \leqq Q /\left(A^{n}|p(z)|\right) \leqq Q /\left(A^{n+1}|z|\right) .
$$

This completes the inductive proof of (12).

We now conclude that $\varphi_{n}(z)$ tends to a limit as $n \rightarrow \infty$, namely

$$
\lim _{n \rightarrow \infty} \varphi_{n}(z)=\varphi_{0}(z)+\sum_{n=1}^{\infty}\left\{\varphi_{n}(z)-\varphi_{n-1}(z)\right\}=\varphi(z)
$$

since the infinite series is dominated by $(Q /|z|) \Sigma A^{-n}<\infty$. The convergence is uniform for $|z| \geqq R$. Therefore, the limit, $\varphi(z)$, is analytic for $|z|>R$. 
It remains to prove that there is no other function, $\psi(z)$, analytic for $|z|>R$, with $\psi(\infty)=0$, such that

$$
\psi(z)=q(z)+\frac{1}{m} \psi(p(z))
$$

Let $\varphi(z)-\psi(z)=w(z)$. Then (9) and (13) imply

$$
w(z)=\frac{1}{m} w(p(z))
$$

If $w(z) \neq 0$, then $w(z) \cong W z^{-N}$ as $z \rightarrow \infty$, where $W \neq 0$ and $N$ is a positive integer. Then (14) implies

$$
W z^{-N} \cong \frac{1}{m} W(p(z))^{-N} \quad \text { as } \quad z \rightarrow \infty
$$

which is absurd, since $m \geqq 1$ and $|p(z) / z| \geqq A>1$. This completes the proof of the theorem.

IV. Application to the quadratic recursion. Keeping $r>0$ fixed, we define $\beta_{n}=\beta_{n}^{(r)}=b_{n}^{(r)}-(r / 2)$. Then (1) implies

$$
\beta_{n+1}=\beta_{n}^{2}-\rho, \quad \text { where } \rho=r(r-2) / 4, \quad n=1,2,3, \cdots
$$

Then $\beta_{n} \rightarrow \infty$ because $\beta_{n} \geqq 1+\frac{1}{2} r$ if $n \geqq 1$, and hence, $\beta_{n+1} \geqq$ $\beta_{n}+r$. Define

$$
p(z)=z^{2}-\rho
$$

Then (15) takes the form

$$
\beta_{n+1}=p\left(\beta_{n}\right) \quad(n=1,2, \cdots)
$$

If $A>1$, then

$$
|p(z)|=\left|z^{2}-\rho\right| \geqq\left|z^{2}\right|-|\rho| \geqq A|z| \text { if } \quad|z| \geqq R
$$

provided that we define

$$
R=\frac{1}{2}\left(A+\left(A^{2}+4|\rho|\right)^{\frac{1}{2}}\right)
$$

since this is the positive root of $R^{2}-|\rho|=A R$.

According to our theorem, there is a unique function of the form 


$$
f(z)=z+c_{0}+\frac{c_{1}}{z}+\cdots(|z|>R)
$$

such that, if $p(z)=z^{2}+\cdots$, then

$$
f(p(z)) \equiv\{f(z)\}^{2} \text { for } \quad(|z|>R) .
$$

Since $p(z)=z^{2}-\rho$ is an even function of $z, f(z)$ must be an odd function of $z$ in order to satisfy (21), and only the $c_{i}$ with odd subscripts need be retained in (20). For all $n \geqq 3$, we have

$$
\beta_{n}>\beta_{2}=1+\frac{3}{2} r
$$

Moreover, since $r>0$,

$$
\begin{aligned}
\beta_{2}^{2}-|\rho| & =1+3 r+\frac{9}{4} r^{2}-\frac{1}{4} r|r-2| \\
>1+\frac{3}{2} r & =\beta_{2} .
\end{aligned}
$$

Define $A$ as any number satisfying

$$
1<A<\frac{\beta_{2}^{2}-|\rho|}{\beta_{2}}
$$

and define $R$ by (19). Then the last inequality implies $\beta_{2}>R$, and hence $\beta_{n}>R$ for all $n \geqq 2$. Hence, for $n \geqq 2$, (17) and (21) imply

$$
f\left(\beta_{n+1}\right)=f\left(p\left(\beta_{n}\right)\right)=\left\{f\left(\beta_{n}\right)\right\}^{2}
$$

Therefore, if $n \geqq 2$,

$$
f\left(\beta_{n}\right)=\theta^{2^{n}}
$$

if $\theta$ is the positive number defined by $f\left(\beta_{2}\right)=\theta^{4}$. Since $\beta_{n} \rightarrow \infty$, we have $\theta>1$.

The continuity of $\theta(r)$. If $r$ ranges in any interval $r_{0} \leqq r \leqq r_{1}$, then there is a constant $A$, independent of $r$, satisfying (22). Then the sequence of iterates $\varphi_{n}(z)$ defined by (10) converges uniformly in $z$ and in $r$, and $\varphi_{n}(z)$ depends continuously on the parameter $r$. Hence, $\lim _{(n \rightarrow \infty)} \varphi_{n}(z)$ is continuous as a function of $r$; and $f(z)$ depends continuously on the parameter $r$. But we have just shown that 


$$
\begin{aligned}
\theta(r) & =\left\{f\left(\beta_{2}\right)\right\}^{1 / 4} \\
& =\left\{f\left(1+\frac{3}{2} r\right)\right\}^{1 / 4}
\end{aligned}
$$

Hence, $\theta(r)$ depends continuously on $r$.

For sufficiently large $|z|$, every function $f(z)$ of the form (20) has an inverse function of the form

$$
F(w)=w+\gamma_{0}+\frac{\gamma_{1}}{w}+\cdots
$$

satisfying

$$
F(f(z)) \equiv z
$$

For the case $p(z)=z^{2}-\rho, f(z)$ is an odd function of $z$, and the inverse function of an odd function is an odd function. Applying $F$ to (23), we find, for all sufficiently large $n$,

$$
\beta_{n}=F\left(\theta^{2^{n}}\right)=\theta^{2^{n}}+\gamma_{1} \theta^{-2^{n}}+\gamma_{3} \theta^{-3 \cdot 2^{n}}+\cdots .
$$

The coefficients in the power series for $f(z)$ and $F(w)$ can be calculated recursively. Formula (21) yields

$$
p(z)+\frac{c_{1}}{p(z)}+\frac{c_{3}}{p^{3}(z)}+\cdots=\left(z+\frac{c_{1}}{z}+\frac{c_{3}}{z^{3}} \cdots\right)^{2}
$$

from which

$$
z^{2}-\rho+\frac{c_{1}}{z^{2}-\rho}+\cdots=z^{2}+2 c_{1}+\left(2 c_{3}+c_{1}^{2}\right) z^{-2}+\cdots
$$

Therefore,

$$
c_{1}=-\frac{\rho}{2}, \quad c_{3}=-\frac{\rho(\rho+2)}{8}, \quad \text { etc. }
$$

For the inverse function, we must have

$$
z \equiv\left(z+c_{1} z^{-1}+c_{3} z^{-3}+\cdots\right)+\gamma_{1}(z+\cdots)^{-1}+\cdots .
$$

Hence 


$$
\gamma_{1}=-c_{1}=\frac{\rho}{2}=\frac{r(r-2)}{8}
$$

$$
\gamma_{3}=-c_{3}-c_{1}^{2}=-\frac{\rho(\rho-2)}{8}=-\frac{(r+2) r(r-2)(r-4)}{128}
$$

etc.

Now (24) yields, for $n \rightarrow \infty$,

$$
\beta_{n}=\theta^{2^{n}}+\frac{1}{8} r(r-2) \theta^{-2^{n}}+O\left(\theta^{-3 \cdot 2^{n}}\right)
$$

If $r=2$ or $r=4$, the preceding sections have shown that $\beta_{n}$ has the exact form

$$
\beta_{n}=\theta^{2^{n}}+\frac{r(r-2)}{8} \theta^{-2^{n}}
$$

and

$$
b_{n}=\theta^{2^{n}}+\frac{r}{2}+\frac{1}{8} r(r-2) \theta^{-2^{n}}
$$

Conversely, it is easily verified that, for $r>0$, and expression of this exact form can satisfy (15) only if $r=2$ or 4 . In fact, the only real values of $\rho$ which lead to this exact form are $\rho=0$ and $\rho=2$.

We remark that the inverse function, $F$, satisfies a functional equation. Letting $z=F(w)$ in the identity

$$
f(p(z))=c\{(z)\}^{m}
$$

yields

$$
f(p(F(w)))=c w^{m} .
$$

Applying $F$ to both sides now yields

$$
p(F(w))=F\left(c w^{m}\right) .
$$

In our application, we have the identity

$$
F^{2}(w)-\rho=F\left(w^{2}\right) .
$$

The coefficients in the power series for $F(w)$ can be calculated directly from this equation. 
The algebraic character of the constant, $\theta$, depends on the nature of the function $f(z)$. If $r$ is a positive integer, then $\beta_{2}$ is an integer or a half-integer, and

$$
\theta=\left\{f\left(\beta_{2}\right)\right\}^{\frac{1}{4}}
$$

Thus, $\theta$ is algebraic if $f(z)$ is algebraic over the field of rationals. In the known cases,

$$
\begin{aligned}
& f(z)=z \quad \text { if } \quad r=2 \\
& f(z)=\frac{1}{2}\left[z+\left(z^{2}-4\right)^{\frac{1}{2}}\right] \quad \text { if } \quad r=4
\end{aligned}
$$

since these are the functions whose inverse functions are

$$
\begin{aligned}
& F(w)=w \quad \text { if } \quad r=2 \\
& F(w)=w+w^{-1} \quad \text { if } \quad r=4 .
\end{aligned}
$$

Unfortunately, only $\rho=0$ and $\rho=2$ lead to functions $F(w)$ with finite expansions in $w$.

V. Coefficient relationships. In the previous section, we considered the functions

$$
f(z)=z+c_{1} z^{-1}+c_{3} z^{-3}+c_{5} z^{-5}+\cdots
$$

and

$$
F(w)=w+\gamma_{1} w^{-1}+\gamma_{3} w^{-3}+\gamma_{5} w^{-5}+\cdots
$$

where

$$
\begin{aligned}
F(f(z)) & \equiv z, \\
f\left(z^{2}-\rho\right) & =f^{2}(z) \quad \text { and } \\
F^{2}(w)-\rho & =F\left(w^{2}\right) .
\end{aligned}
$$

In this section, we shall assume only that $\rho$ is a real number, and derive certain identities involving the coefficient sequences $\left\{c_{i}\right\}$ and $\left\{\gamma_{i}\right\}$.

From (29) and (31), we obtain

$$
\sum_{\text {odd } k=-1}^{\infty} \frac{c_{k} u^{k}}{(1-\rho u)^{k}}=\sum_{m=-1}^{\infty} \sum_{\text {odd } j=-1}^{2 m+1} c_{j} c_{2 m-j} u^{m}
$$

where for convenience we have set $c_{-1}=1$. 
We rewrite (30) using $t=w^{-1}$ and $q_{i}=\gamma_{2 i-1}$ to obtain

$$
F(1 / t)=\frac{1}{t}+q_{1} t+q_{2} t^{3}+q_{3} t^{5}+q_{4} t^{7}+\cdots=\sum_{i=0}^{\infty} q_{i} t^{2 i-1}
$$

where we have set $q_{0}=1$.

Then from (31) we find

$$
\begin{array}{ll}
q_{1}=\frac{\rho}{2} & q_{5}=-q_{1} q_{4}-q_{2} q_{3} \\
q_{2}=-\left(\begin{array}{c}
q_{1} \\
2
\end{array}\right) & q_{6}=-q_{1} q_{5}-q_{2} q_{4}-\left(\begin{array}{c}
q_{3} \\
2
\end{array}\right) \\
q_{3}=-q_{1} q_{2} & q_{7}=-q_{1} q_{6}-q_{2} q_{5}-q_{3} q_{4} \\
q_{4}=-q_{1} q_{3}-\left(\begin{array}{c}
q_{2} \\
2
\end{array}\right) & q_{8}=-q_{1} q_{7}-q_{2} q_{6}-q_{3} q_{5}-\left(\begin{array}{c}
q_{4} \\
2
\end{array}\right)
\end{array}
$$

and in general

$$
q_{2 l-1}=-\sum_{i=1}^{1-1} q_{i} q_{2 l-1-i}, \quad l>1
$$

$$
q_{2 l}=-\left(\begin{array}{c}
q_{l} \\
2
\end{array}\right)-\sum_{i=1}^{l-1} q_{i} q_{2 l-i}, \quad l \geqq 1
$$

The assertion that the expansion for $F(w)$ is finite only in the cases $\rho=0$ and $\rho=2$ is proved as follows. Suppose

$$
F(w)=w+\frac{q_{1}}{w}+\frac{q_{2}}{w^{3}}+\cdots+\frac{q_{n}}{w^{2 n-1}}, \quad n>1,
$$

where $q_{n}$ is the last nonzero coefficient. From $F\left(w^{2}\right)+\rho=F^{2}(w)$, we see that $q_{n}^{2}=q_{n}$, so that $q_{n}=1$. Next, $q_{n-1}=0$, because $2 q_{n-1}$ is the coefficient of $w^{-(4 n-4)}$ in $F^{2}(w)$, but the corresponding coefficient in $F\left(w^{2}\right)$ is 0 . The next coefficient identity, examining $w^{(4 n-6)}$. is $q_{n-1}=$ $2 q_{n-2}+q_{n-1}^{2}$, but since $q_{n-1}=0$ we get $q_{n-2}=0$, and proceeding inductively, we find $q_{i}=0$ for all $i, 1 \leqq i \leqq n-1$. However, $F(w)=$ $w+1 / w^{2 n-1}$ fails to satisfy the functional equation for all $n>1$. The cases $F(w)=w$ and $F(w)=w+1 / w$ are the unique solutions of the functional equation for $n=0$ and $n=1$ respectively.

Other than for $\rho=0$ and $\rho=2$, no cases of "well-known" functions have been found as either $f(z)$ or $F(w)$. However, the case $\rho=-2$ 
turns out to be of considerable combinatorial interest. The recursion equations (34), starting with $\rho=-2$ and $q_{1}=-1$, generate a sequence of integers $\left\{q_{1}\right\}$ such that $\left\{-q_{1}\right\}=\{1,1,1,2,3,6,11,23,46,98, \cdots\}$, where it is easily seen (1) that $-q_{t}$ is the number of distinct "binary coding trees" with $i=1$ interior nodes and $i$ terminal nodes, where there are always two edges leading down from each interior node (see Figure (1); and (2) that $-q_{i}$ is the number of binary rooted trees with $i$ nodes altogether, and at most two edges leading down from each node (see Figure 2). Indeed, the second set of trees is obtained by pruning away all branches leading to terminal nodes in the first set of trees. A recursion strikingly similar to (34) occurs in yet another tree enumeration problem [2].

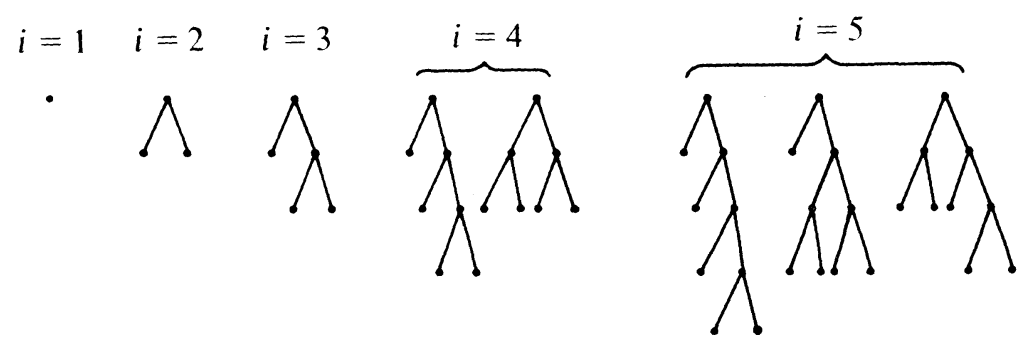

$i=6$
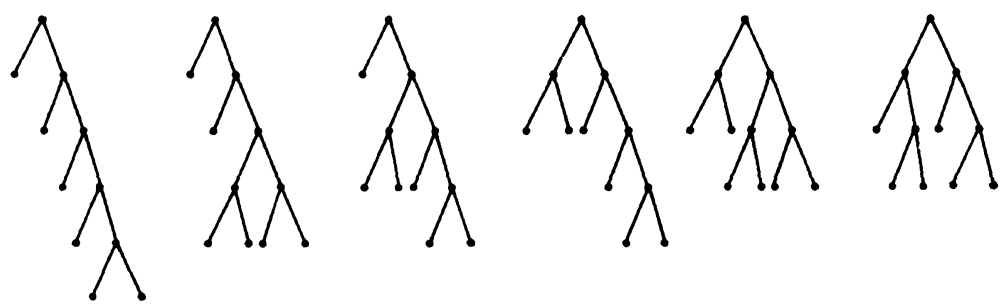

$i=7$

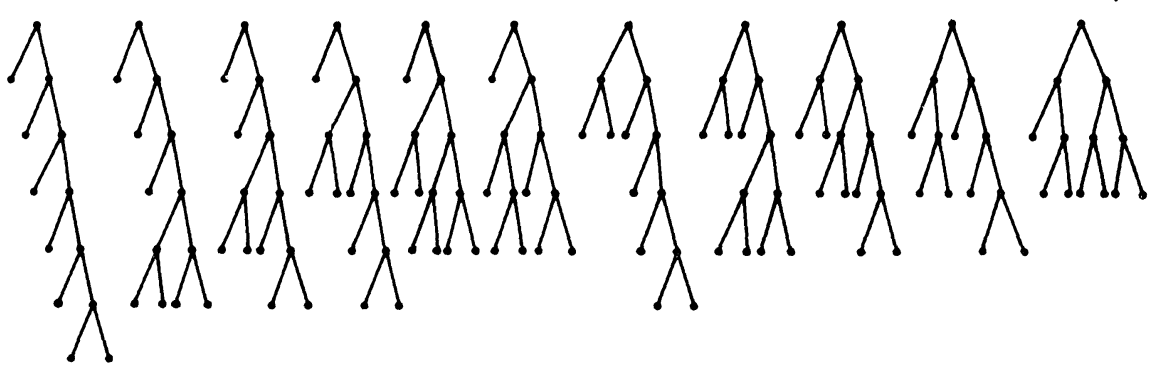

FIGURE 1. The "binary coding trees" of order $i, 1 \leqq i \leqq 7$. 


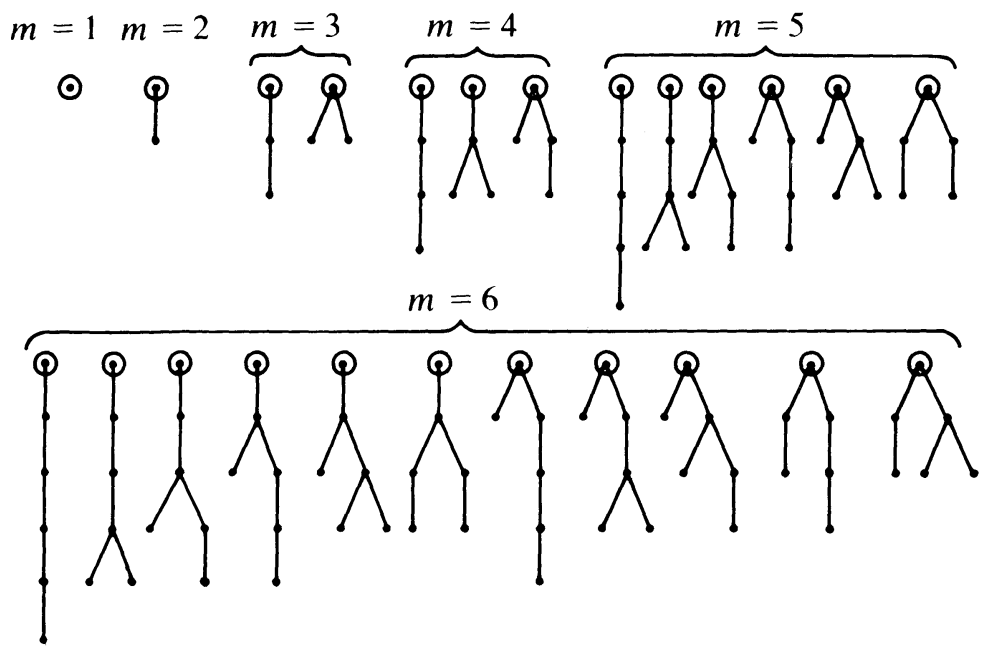

$m=7$

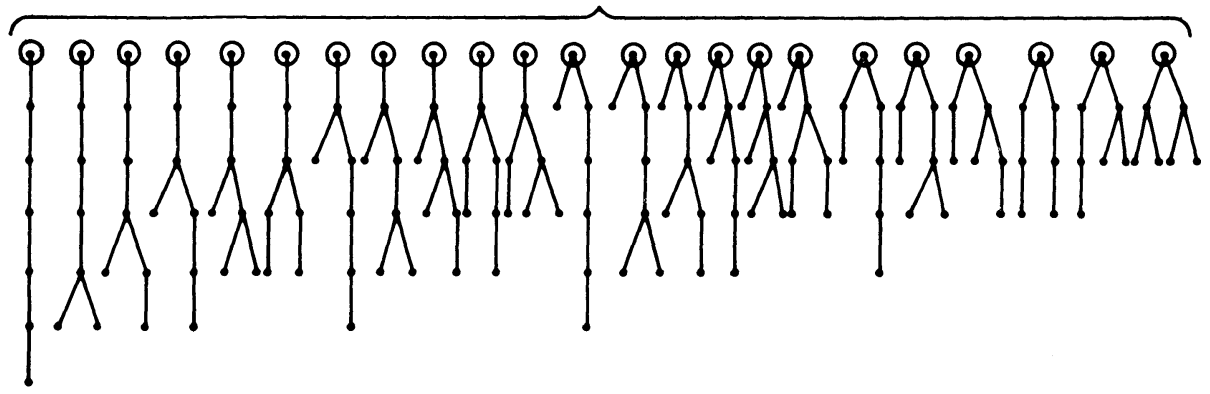

FIGURE 2. The "binary rooted trees" with $m=i-1$ nodes, $1 \leqq m \leqq 7$.

Let $f(z)$ satisfy (29) and (31). Then equation (32) can be solved to obtain the following recursion relation for the coefficient sequence $\left\{c_{2 i+1}\right\}$, starting with $c_{-1}=1$ :

$$
2 c_{2 \imath+1}=-\sum_{\text {odd } j=1}^{2 i-1} c_{j} c_{2 i-j}+\sum_{\text {odd } j=1}^{l} c_{l}\left(\begin{array}{l}
i-1 \\
j-1
\end{array}\right) \rho^{i-j}
$$

Thus, if $i$ even,

$$
c_{2 i+1}=-\sum_{\text {odd } j=1}^{i-1} c_{j} c_{2 i-j}+\frac{1}{2} \sum_{\text {odd } j=1}^{i-1} c_{j}\left(\begin{array}{l}
i-1 \\
j-1
\end{array}\right) \rho^{i-j}
$$

while if $i$ odd,

$$
c_{2 i+1}=-\sum_{\text {odd } j=1}^{i-2} c_{j} c_{2 i-j}-\left(\begin{array}{c}
c_{i} \\
2
\end{array}\right)+\frac{1}{2} \sum_{\text {odd } j=1}^{i-2} c_{j}\left(\begin{array}{l}
i-1 \\
j-1
\end{array}\right) \rho^{i-j}
$$


Equivalently:

$$
2 c_{2 i+1}=-\sum_{\text {odd } j=1}^{2 i-1} c_{j} c_{21-j}+\sum_{\substack{m=0 \\
(i-m \text { odd })}}^{i-1}\left(\begin{array}{c}
i-1 \\
m
\end{array}\right) c_{i-m} \rho^{m} .
$$

Thus, if $i$ even,

$$
c_{2 i+1}=-\sum_{\text {odd } j=1}^{i-1} \mid c_{j} c_{2 i-j}+\frac{1}{2} \sum_{\text {odd } m=1}^{i-1}\left(\begin{array}{c}
i-1 \\
m
\end{array}\right) c_{i-m} \rho^{m},
$$

while if $i$ odd,

$$
c_{2 i+1}=-\sum_{\text {odd } j=1}^{i-2} c_{j} c_{2 i-j}-\left(\begin{array}{c}
c_{i} \\
2
\end{array}\right)+\frac{1}{2} \sum_{\text {even } m=2}^{i-1}\left(\begin{array}{c}
i-1 \\
m
\end{array}\right) c_{i-m} \rho^{m} .
$$

Explicitly:

$$
\begin{aligned}
& c_{1}=-\frac{\rho}{2} \\
& c_{3}=-\left(\begin{array}{c}
c_{1} \\
2
\end{array}\right) \\
& c_{5}=-c_{1} c_{3}+\frac{c_{1} \rho}{2} \\
& c_{7}=-c_{1} c_{5}-\left(\frac{c_{3}}{2}\right)+\frac{c_{1} \rho^{2}}{2}
\end{aligned}
$$

(42) $c_{9}=-c_{1} c_{7}-c_{3} c_{5}+\frac{c_{1} \rho^{3}+3 c_{3} \rho}{2}$

$$
\begin{aligned}
& c_{11}=-c_{1} c_{9}-c_{3} c_{7}-\left(\frac{c_{5}}{2}\right)+\frac{c_{1} \rho^{4}+6 c_{3} \rho^{2}}{2} \\
& c_{13}=-c_{1} c_{11}-c_{3} c_{9}-c_{5} c_{7}+\frac{c_{1} \rho^{5}+10 c_{3} \rho^{3}+5 c_{5} \rho}{2} \\
& c_{15}=-c_{1} c_{13}-c_{3} c_{11}-c_{5} c_{9}-\left(\begin{array}{c}
c_{7} \\
2
\end{array}\right)+\frac{c_{1} \rho^{6}+15 c_{3} \rho^{4}+15 c_{5} \rho^{2}}{2} \\
& c_{17}=-c_{1} c_{15}-c_{3} c_{13}-c_{5} c_{11}-c_{7} c_{9}+\frac{c_{1} \rho^{7}+21 c_{3} \rho^{5}+35 c_{5} \rho^{3}+7 c_{7} \rho}{2}
\end{aligned}
$$

etc. 
VI. Asymptotic ratios. Define $G(u)$ from (33) by

$$
G(u)=u^{\frac{1}{2}} F\left(u^{-\frac{1}{2}}\right)=1+q_{1} u+q_{2} u^{2}+q_{3} u^{3}+q_{4} u^{4}+\cdots=\sum_{i=0}^{\infty} q_{i} u^{i}
$$

Suppose there is a limiting ratio $R=\lim _{i \rightarrow \infty}\left(q_{i+1} / q_{i}\right)$. Then it is well known that the radius of convergence of the power series (43) for $G(u)$ is $1 /|R|$. Therefore the series (30) for $F(w)$ converges for $|w|>\left|R^{\frac{1}{2}}\right|$, diverges for $|w|<\left|R^{\frac{1}{2}}\right|$, and may converge for some (or all) of the values of $w$ with $|w|=\left|R^{\frac{1}{2}}\right|$. By (34), if $\rho$ is real, then all the $q_{i}$ are real, and $R$ is real, $R= \pm|R|$.

From (34) we have

$$
-q_{m+1}=q_{1} q_{m}+q_{2} q_{m-1}+q_{3} q_{m-2}+\cdots,
$$

and therefore, for large $m$, dividing by $q_{m}$ yields

$$
-R=q_{1}+\frac{q_{2}}{R}+\frac{q_{3}}{R^{2}}+\frac{q_{4}}{R^{3}}+\cdots
$$

and in view of (30), $R$ satisfies

$$
R+q_{1}+\frac{q_{2}}{R}+\frac{q_{3}}{R^{2}}+\frac{q_{4}}{R^{3}}+\cdots=R^{\frac{1}{2}} F\left(R^{\frac{1}{2}}\right)=0,
$$

provided that $R^{\frac{1}{2}}$ is a point of convergence of the series (30) for $F(w)$. In this case, invoking (31), we find:

$$
\begin{gathered}
F(R)=F^{2}\left(R^{\frac{1}{2}}\right)-\rho=-\rho \\
R=f(-\rho)=-f(\rho) .
\end{gathered}
$$

It would be interesting to determine the domain of values of $R$ for which (45) holds. Within that domain, (45) can be used in the practical computation of $R$. For example, when $\rho=-2$ in (42), we find:

$$
\begin{gathered}
c_{1}=1, \quad c_{3}=0, \quad c_{5}=-1, \quad c_{7}=1, \quad c_{9}=-5, \quad c_{11}=12, \\
c_{13}=-22, \quad c_{15}=19, \quad c_{17}=68, \cdots
\end{gathered}
$$

The limiting ratio $R$ of the sequence

$\left\{-q_{i}\right\}=\{1,1,1,2,3,6,11,23,46,98,207,451,983,2179,4850,10905, \cdots\}$ 
of the number of binary coding trees is then given by (45) as

$$
R=f(2)=2+\frac{1}{2}-\frac{1}{32}+\frac{1}{127}-\frac{5}{512}+\frac{12}{2048}-\frac{22}{8192} \pm \cdots=2 \cdot 470 \cdots
$$

which is in good agreement with the empirical value.

From (34), for $\rho<0$, all the $q_{\text {i }}$ 's are negative, while for $\rho>2$, the

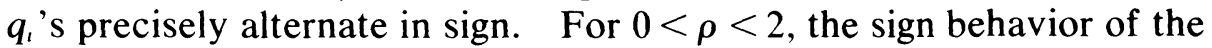
$q_{i}$ 's is considerably more intricate, and is not the same throughout this interval. The relation $F^{2}(w)-\rho=F\left(w^{2}\right)$ is sufficient to insure the convergence of $F(w)$ at $w=\left|R^{\frac{1}{2}}\right|$ provided that $|R|<1$ and that the $q_{\text {i }}$ 's become and remain, for large $i$, either all of the same sign, or strictly alternating in sign. This sufficient condition can be restated entirely in terms of the value of $\rho: F(w)$ must converge at $w=\left|R^{\frac{1}{2}}\right|$ for all $\rho<\rho_{1} \approx-0.43$, and for all $\rho>\rho_{2}=2$. Whether this condition is also necessary has not been determined.

ACKNOWLEDGMENTS. We wish to acknowledge a timely communication from D. Knuth containing an explicit solution for the case $r=4$, which rekindled our interest in this topic. A recent article [3] also discusses the class of sequence in [1].

\section{REFERENCES}

1. S. Golomb, On certain nonlinear recurring sequences, Amer. Math. Monthly, 70, no. 4, (April, 1963), 403-405.

2. Clifford W. Marshall, Applied Graph Theory, Wiley, (1971), 172-173.

3. A. V. Aho, and N. J. A. Sloane, Some doubly exponential sequences, The Fibonacci Quarterly, 11, no. 4 (Nov., 1973), 429-437.

Received March 7, 1974. The research of Dr. J. N. Franklin, of the California Institute of Technology, was supported in part by AEC Contract AT(04-3)-767, Project Agreement \# 12. The research of Dr. S. W. Golomb, of the University of Southern California, was supported in part by the U.S.Army Research Office, under Contract DA-ARO-D-31-124-73-G167.

UNIVERSITY OF SOUTHERN CALIFORNIA 


\section{PACIFIC JOURNAL OF MATHEMATICS \\ EDITORS}

\author{
Richard ARENS (Managing Editor) \\ University of California \\ Los Angeles, California 90024
}

\author{
R. A. Beaumont \\ University of Washington \\ Seattle, Washington 98105
}

\section{J. DugundJI}

Department of Mathematics

University of Southern California

Los Angeles, California 90007

D. Gilbarg and J. Milgram

Stanford University

Stanford, California 94305

\section{ASSOCIATE EDITORS}
E. F. BECKENBACH
B. H. NeumanN
F. WOLF
K. YoshidA

\section{SUPPORTING INSTITUTIONS}

\author{
UNIVERSITY OF BRITISH COLUMBIA \\ CALIFORNIA INSTITUTE OF TECHNOLOGY \\ UNIVERSITY OF CALIFORNIA \\ MONTANA STATE UNIVERSITY \\ UNIVERSITY OF NEVADA \\ NEW MEXICO STATE UNIVERSITY \\ OREGON STATE UNIVERSITY \\ UNIVERSITY OF OREGON \\ OSAKA UNIVERSITY
}

\author{
UNIVERSITY OF SOUTHERN CALIFORNIA \\ STANFORD UNIVERSITY \\ UNIVERSITY OF TOKYO \\ UNIVERSITY OF UTAH \\ WASHINGTON STATE UNIVERSITY \\ UNIVERSITY OF WASHINGTON \\ AMERICAN MATHEMATICAL SOCIETY
}

The Supporting Institutions listed above contribute to the cost of publication of this Journal, but they are not owners or publishers and have no responsibility for its contents or policies.

Mathematical papers intended for publication in the Pacific Journal of Mathematics should be in typed form or offset-reproduced (not dittoed), double spaced with large margins. Underline Greek letters in red, German in green, and script in blue. The first paragraph or two must be capable of being used separately as a synopsis of the entire paper. Items of the bibliography should not be cited there unless absolutely necessary, in which case they must be identified by author and Journal, rather than by item number. Manuscripts, in duplicate, may be sent to any one of the four editors. Please classify according to the scheme of Math. Reviews, Index to Vol. 39. All other communications should be addressed to the managing editor, or Elaine Barth, University of California, Los Angeles, California, 90024.

100 reprints are provided free for each article, only if page charges have been substantially paid. Additional copies may be obtained at cost in multiples of 50 .

The Pacific Journal of Mathematics is issued monthly as of January 1966. Regular subscription rate: $\$ 72.00$ a year (6 Vols., 12 issues). Special rate: $\$ 36.00$ a year to individual members of supporting institutions.

Subscriptions, orders for back numbers, and changes of address should be sent to Pacific Journal of Mathematics, 103 Highland Boulevard, Berkeley, California, 94708.

PUBLISHED BY PACIFIC JOURNAL OF MATHEMATICS, A NON-PROFIT CORPORATION Printed at Jerusalem Academic Press, POB 2390, Jerusalem, Israel.

$$
\begin{gathered}
\text { Copyright (c) } 1975 \text { Pacific Journal of Mathematics } \\
\text { All Rights Reserved }
\end{gathered}
$$




\section{Pacific Journal of Mathematics}

Vol. 56, No. 2 December, 1975

Ralph Alexander, Generalized sums of distances .................. 297

Zvi Arad and George Isaac Glauberman, A characteristic subgroup of a group of odd order ............................... 305

B. Aupetit, Continuité du spectre dans les algèbres de Banach avec involution ........................................ 321

Roger W. Barnard and John Lawson Lewis, Coefficient bounds for some classes of starlike functions . ...........................

Roger W. Barnard and John Lawson Lewis, Subordination theorems for some classes of starlike fumctions . .................... 333

Ladislav Bican, Preradicals and injectivity .................. 367

James Donnell Buckholtz and Ken Shaw, Series expansions of analytic

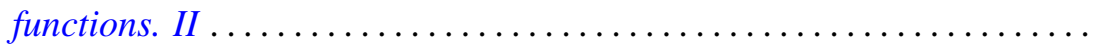

Richard D. Carmichael and E. O. Milton, Distributional boundary values in

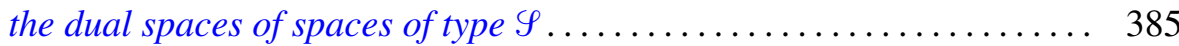

Edwin Duda, Weak-unicoherence ............................ 423

Albert Edrei, The Padé table of functions having a finite number of essential singularities ........................................

Joel N. Franklin and Solomon Wolf Golomb, A function-theoretic approach to the study of nonlinear recurring sequences ................ 455

George Isaac Glauberman, On Burnside's other $p^{a} q^{b}$ theorem 469

Arthur D. Grainger, Invariant subspaces of compact operators on topological vector spaces .........................

Jon Craig Helton, Mutual existence of sum and product integrals .

Franklin Takashi Iha, On boundary functionals and operators with finite-dimensional null spaces

Gerald J. Janusz, Generators for the Schur group of local and global number fields

A. Katsaras and Dar-Biau Liu, Integral representations of weakly compact operators.

W. J. Kim, On the first and the second conjugate points .

Charles Philip Lanski, Regularity and quotients in rings with involution ....

Ewing L. Lusk, An obstruction to extending isotopies of piecewise linear manifolds.

Saburou Saitoh, On some completenesses of the Bergman kernel and the Rudin kernel..

Stephen Jeffrey Willson, The converse to the Smith theorem for $Z_{p}$-homology spheres. 